\title{
Stem cells in gastrointestinal cancers
}

\author{
Aiwu Ruth He ${ }^{\mathrm{a}, *}$, Jonathan Mendelson ${ }^{\mathrm{b}}$, Tiffany Blake ${ }^{\mathrm{b}}$, Lopa Mishra ${ }^{\mathrm{b}, \mathrm{c}}$ and John L. Marshall ${ }^{\mathrm{a}}$ \\ ${ }^{a}$ Division of Hematology/Oncology, Lombardi Comprehensive Cancer Center, Georgetown University, Washington, \\ $D C, U S A$ \\ ${ }^{\mathrm{b}}$ Laboratory of Cancer Genetics, Digestive Diseases, and Developmental Molecular Biology, Department of \\ Surgery, Lombardi Cancer Center, Georgetown University, Washington, DC, USA \\ ${ }^{\mathrm{c}}$ Laboratory of Digestive Diseases, Department of Surgery, DVAMC, Washington, DC, USA
}

\section{Introduction}

The malignant change from normal to cancer stem cells is a hallmark transition in gastro-intestinal carcinogenesis. In addition to their ability for self-renewal, stem cells are sufficiently long-lived to acquire the necessary sequential mutations that allow for malignant transformation. Cancer stem cells (CSCs) have the capacity to initate and maintain tumor growth in several cancers, though only in the past decade have these cells been identified and characterized in hematological malignancies [1-4]. Recent studies have described CSCs in solid tumors including cancer of the breast, prostate, brain, colon, pancreas, and liver [5-21]. However the mechanism underlying the emergence of cancer stem cell formation remains elusive. In GI cancers, signaling pathways such as TGF- $\beta$, Wnt, FGFs, and Hedgehog are responsible for gut development and have a potential role in the formation of CSCs. Underlying this hypothesis is the finding that deregulated signaling pathways in gut development, as manifested in rare human cancers as well as genetic mouse studies, are driven by a population of CSCs. Importantly, it was found that the same surface markers used to identify embryonic stem cells are capable of identifying and sorting CSCs as well. These phenomena point to the need for scientists to understand how CSCs become insensitive to

\footnotetext{
*Corresponding author: Aiwu Ruth He, Laboratory of Developmental Biology/Digestive Diseases, Georgetown University, Medical/Dental Building, NW 213, 3900 Reservoir Road, N.W., Washington, DC 20007, USA. E-mail: arh29@gunet.georgetown.edu.
}

inhibitory signals as well as chemotherapeutic agents and are thus permitted to continuously self-renew. Targeting CSCs holds much promise for the development of novel therapeutic agents.

\section{Identification of the key genes in cancer stem cells of GI malignancies}

\subsection{Role of Wnt/ $\beta$-Catenin signaling pathway}

Mutations in the adenomatous polyposis coli (APC) gene, a critical component of the WNT pathway, act to suppress Wnt signaling and result in familial adenomatous polyposis (FAP) syndrome [22]. In the canonical Wnt pathway, the binding of Wnt ligands to the Fz receptors results in activation of the disheveled protein and the subsequent inhibition of glycogen synthase kinase $3 \beta$ (GSK-3 $\beta$ ). This in turn prevents APC and Axin dependent degradation of $\beta$-catenin, leading to its accumulation in the cytosol, where it then translocates to the nucleus and binds to transcription factors that regulate tissue patterning, cell fate, and cell proliferation [23]. The canonical Wnt $/ \beta$-catenin signaling pathway plays a central role in modulating the delicate balance between self-renewal and differentiation in several adult stem cell niches including regeneratioin of the mammary gland, hair follicle, intestinal crypt, and the skin $[24,25]$. In the majority of sporadic colorectal cancer cases, either loss of $A P C$ function or oncogenic $\beta$ catenin mutations seem to be the early events in tumor development. Apc1638N, the chain-termination muta- 
tion, results in multiple intestinal tumors in mice [26]. Likewise, mutations in the glycogen synthase kinase $3 \beta$ (GSK-3 $\beta$ ) phosphorylation sites of the $\beta$-catenin gene are found in 20-30\% of human primary hepatocellular carcinoma (HCC) [27], while mutations in the APC or AXIN genes are found in other HCC populations [28]. These findings point to the canonical Wnt cascade as a critical regulator of stem cells, and highlight the importance of accumulated nuclear $\beta$-catenin as a key event in carcinogenesis of GI malignancies [29].

\subsection{Role of BMP receptor $1 A$ and TGF $\beta$ family signaling}

The TGF- $\beta$ family signaling is most prominent at the interface of development and cancer in gut epithelial cells and is a key player in the self-renewal and maintenance of stem cells [30]. TGF- $\beta$ forms a complex with the serine-threonine kinase receptors type I (T $\beta \mathrm{RI})$ and II (T $\beta$ RII). The constitutively active T $\beta$ RII phosphorylates $\mathrm{T} \beta \mathrm{RI}$, which in turn phosphorylates one of the receptor-activated (R-Smads) [31]. The active R-Smad will heterodimerize with the common mediator Smad, Smad4, and the two translocate to the nucleus where they drive the transcription of target genes. Their activity is modulated by adaptors such as SARA in the case of Smad2 [32], and ELF in the case of Smad3 and Smad4 [33], though the activity of these adaptor proteins also include functional interactions with multiple signal transduction pathways apart from the TGF$\beta$ pathway. When TGF- $\beta$ signaling is disrupted, the imbalance can result in an undifferentiated phenotype which may set the stage for cancer development. Functional breakdown of different TGF- $\beta$ members is observed throughout the spectrum of GI malignancies. For example, colon cancer involves inactivating mutations in the TGF- $\beta$ type II (TBRII) receptor. Moreover, intracellular signaling is disrupted in pancreatic carcinoma through the inactivation of Smad4, also known as DPC4 (deleted in pancreatic carcinoma locus 4), which occurs in one-half of pancreatic carcinoma cases [34]. Significant loss of ELF and reduced Smad4 expression are also found in human gastric and colon cancer tissue samples $[35,36]$. Genetic studies in mice have suggested that loss of TGF- $\beta$ signaling plays an important role during early tumor development. Mice that are heterozygously null for smad4 develop gastric polyps that can develop into tumors at a late age. A wide range of GI tumors, including those of the stomach, liver and

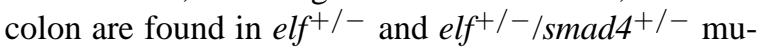
tant mice [37]. Forty percent of $e l f^{+/-}$mice sponta- neously developed hepatocellular carcinoma indicating that functional TGF- $\beta$ signaling is a critical component in maintaining normal stem cells in GI malignancies.

An important finding in spontaneously developed hepatocellular carcinoma involves the observation that CDK4 and IL6/Stat3 signaling are constitutively activated in this cancer. Down-regulating CDK4 or IL6/Stat3 attenuates hepatocellular carcinoma formation. Since CDK4 and IL6/Stat3 play important roles in liver development and regeneration, regulating CDK4 and IL6/Stat 3 signaling by TGF- $\beta$ may be one of the mechanisms responsible for controlling the signal between self-renewal and differentiation in liver stem cells. Mutations in the TGF- $\beta$ pathway, resulting in unregulated CDK4 and IL6/Stat3 activation, lead to increased cell self-renewal and decreased differentiation.

\subsection{Role of PTEN-Akt pathway}

Mutations that affect receptor tyrosine kinase signaling pathways have been found in inherited polyposis syndromes. Mutations in PTEN, a phosphatase that antagonizes PI3 kinase activity, causes Cowden's syndrome which includes hamartomas in the gastrointestinal tract, central nervous system, and skin, as well as tumors of the breast and thyroid gland [38]. Intestinal polyposis, a precancerous neoplasia, results primarily from an abnormal increase in the number of crypts, which contain intestinal stem cells. In PTEN-deficient mice, excess intestinal stem cells initiate de novo crypt formation and crypt fission, recapitulating crypt production in fetal and neonatal intestines [39]. Additionally, PTEN helps control the proliferative rate and the number of intestinal stem cells, and loss of PTEN results in an excess number of intestinal stem cells. It is proposed that the PTEN-Akt pathway probably governs stem cell activation by helping control nuclear localization of the Wnt pathway effector $\beta$-catenin. Nuclear localization of $\beta$-catenin is considered a key event in the activation of stem cells and is potentiated by Akt phosphorylated $\beta$-catenin at Ser552. In addition, Akt phosphorylates GSK-3 $\beta$ at serine 9 , allowing for the accumulation of $\beta$-catenin in the nucleus. This process is found to be Smad7-dependent and regulated by TGF- $\beta$ signaling.

\subsection{Crosstalk among Wnt signaling, TGF- $\beta$ signaling and PTEN-AKT signaling}

Wnt signaling, TGF- $\beta$ signaling, and PTEN-AKT signaling seemingly integrate in the formation of can- 


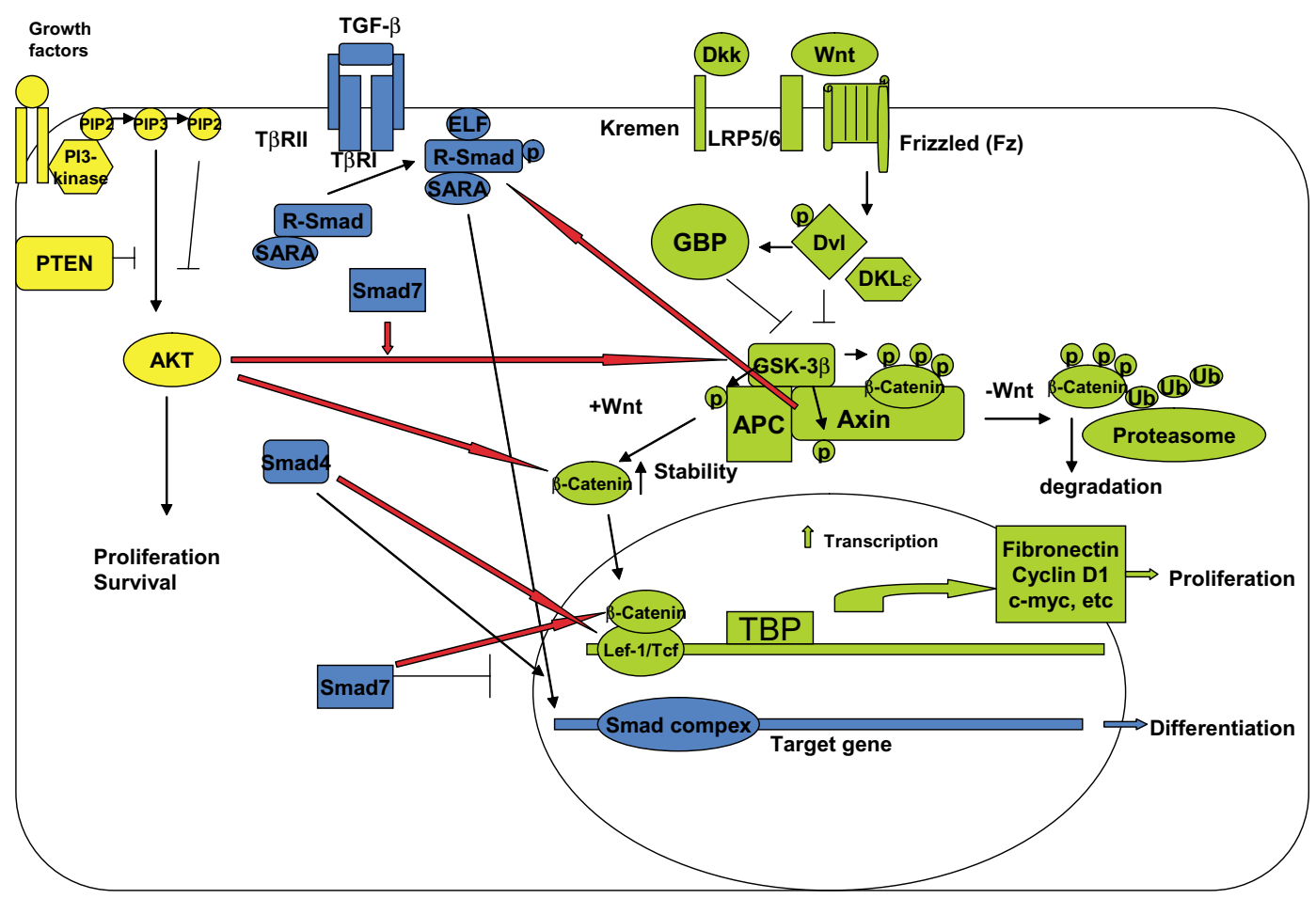

Fig. 1. Crosstalk between Wnt, TGF-b, PTEN-AKT signaling pathway.

cer stem cells in GI malignancies (Fig. 1). As mentioned earlier, PTEN-AKT phosphorylates $\beta$-catenin and GSK-3 $\beta, \beta$-catenin accumulates in the nucleus, and cells are able to proliferate. This crosstalk between PTEN-Akt and Wnt- $\beta$-catenin is Smad7-dependent and TGF- $\beta$ regulated.

Wnt signaling and TGF- $\beta$ signaling interact at different levels. E-cadherin accumulation at cell-cell contacts and E-cadherin- $\beta$-catenin-dependent epithelial cell - cell adhesion is disrupted in $\mathrm{elf}^{+/-} / \mathrm{Smad}^{+/-}$ mutant gastric epithelial cells, though it is rescued by ectopic expression of full-length elf, but not by Smad3 or Smad4 [37]. Smad4 potentiates the activity of transcription factor Lef- 1 downstream $\beta$-catenin [40]. Axin from the Wnt signaling pathway associates with Smad3 in the cytoplasm and facilitates phosphorylation by $\mathrm{T} \beta \mathrm{RI} / \mathrm{II}$, then disassociates when phospho-Smad3 associates with Smad4 [41]. Remarkably, TGF- $\beta$ induced redistribution of $\beta$-catenin is Smad7-dependent [42]. The critical role of $\mathrm{Wnt} / \beta$-Catenin, TGF- $\beta$ family signaling and PTEN-Akt pathways in gut development and GI malignancies already reveal crosstalk possibilities among these pathways which may elucidate the disruption of normal stem cells in GI carcinogenesis, paving the way for future therapeutic development.

\section{Isolation of CSCs}

Multiple surface markers that are used to identify embryonic stem cells have been used to identify and sort cancer stem cells. CD133 (AC133) is a highly conserved antigen as the human homologue of mouse Prominin-1, which was originally identified as a 5 transmembrane cell surface glycoprotein expressed in a subpopulation of $\mathrm{CD}_{3} 4^{+}$hematopoietic stem and progenitor cells derived from human fetal liver and bone marrow [43]. CD133 is expressed by normal primitive cells of the neural, hematopoietic, epithelial and endothelial lineages. Notably, CD133 ${ }^{+}$cells were found in some types of tumor tissues including tumors associated with AML, brain, ependymoma and prostate $[17$, $18,44]$. In glioblastoma as few as $100 \mathrm{CD} 133^{+}$cells were described to be able to produce tumors in immunodeficient mice, whereas $1 \times 10^{5}$ cells from the same tumor without this surface molecule failed to produce a similar outcome [18]. Moreover, CD133 is found to be able to distinguish CSCs from non-CSCs in colon cancer $[45,46]$. In colon cancer it was found that only $2.5 \%$ of tumor cells were in fact CD133+, though only this cohort of cells was capable of reproducing the primary tumor in immuodeficient mice upon subcutaneous injection of these cells. Unlike CD133- cells, CD133+ 
Table 1

Overview of key genes, pathways and surface markers for cancer stem cells in GI malignancies

\begin{tabular}{ll}
\hline & Cancer stem cells in GI malignancies \\
\hline Key genes and Pathways Wnt/ $\beta$-catenin & Loss of APC, accumulation of $\beta$-catenin, mutation of glycogen synthase kinase $3 \beta$ \\
TGF- $\beta$ /BMP signaling & Loss or mutation of TGF $\beta$ type II receptor, Smad4, ELF \\
PTEN-Akt & Loss or mutation of PTEN, activated Akt \\
CDK4 & Activated \\
IL6/Stat3 & Activated \\
Key surface markers & \\
CD133 & Colorectal Cancer, Hepatocellular Carcinoma \\
CD44 & Pancreatic Cancer \\
CD24 & Pancreatic Cancer \\
\hline
\end{tabular}

colon cancer cells grew exponentially for more than one year in vitro as undifferentiated tumor spheres in serum-free medium, maintaining the ability to engraft and reproduce the same morphological and antigenic pattern as the original tumor. $\mathrm{CD}_{133^{+}}$colon cancer cells can differentiate and become $\mathrm{CK} 20^{+}$, thus losing their ability to be transplanted into SCID mice. Following a similar pattern as observed in mice, CSCs from human colon cancer samples may be isolated on the basis of their ability to initiate human colon cancer after transplantation into NOD/SCID mice. Purification experiments established that all CSCs able to initiate

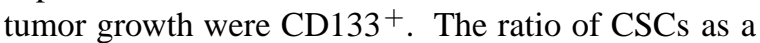
proportion of total tumor cells reveals that there is only one $\mathrm{CD} 133^{+} \mathrm{CSC}$ in $5.7 \times 10^{4}$ unfractionated tumor cells, whereas there is one CD133 ${ }^{+} \mathrm{CSC}$ in only 262 CD133 cells, which represents a $>200$-fold enrichment by using CD133 as a marker. Furthermore, it was observed that CSCs are bidirectional within the CD133+ population, as they were both able to maintain themselves as well as to differentiate and re-establish tumor heterogeneity upon serial transplantation.

The fact that regenerative $\mathrm{CD} 117^{+} / \mathrm{CD} 133^{+}$hepatic precursor cells are identified in fresh frozen liver samples from patients suffering from massive liver necrosis supports the use of CD133 as a marker for liver progenitor cells [47]. CD133 has been used to isolate CSCs in hepatocellular carcinoma recently $[39,48]$. From the SMMC-7721 cell line, CD133 ${ }^{+}$cells isolated by MACS manifested high tumorigenecity and clonogenicity as compared with CD133- ${ }^{-}$HCC cells [46].

However, it is not clear that CD133 is sufficient alone in isolating HCC cancer stem cells. While CD133 is used to sort these progenitors from Huh7 cells, CD133 ${ }^{+}$ cells were detected in $46.7 \%$ of Huh- 7 cells [48]. However, when flow cytometry and the DNA-binding dye Hoechst 33342 were used to isolate side population (SP) cells from various human gastrointestinal system cancer cell lines, SP cells were detected only in $0.25 \%$ of Huh7 [49]. SP analysis and sorting followed by se- rial transplantation of the cells into NOD/SCID mice was used to isolate HCC cells with stem cell properties. Only $1 \times 10^{3} \mathrm{SP}$ cells were sufficient for tumor formation, whereas an injection of $1 \times 10^{6}$ non-SP cells did not initiate tumors. Microarray analysis identified a differential gene expression profile between SP and non-SP cells. It seems two or more markers are necessary to identify CSCs reliably in HCC.

CD44 and CD24 have been used to isolate breast CSCs (Ponti, 2005). A subpopulation of pancreatic cancer cells expressing the cell surface markers CD44, CD24, and epithelial-specific antigen [50] are found to be highly tumorigenic using a xenograft model [51]. Pancreatic cancer cells with the $\mathrm{CD} 44{ }^{+} \mathrm{CD} 24{ }^{+} \mathrm{ESA}^{+}$ phenotype (0.2-0.8\% of pancreatic cancer cells) have a 100-fold increased tumorigenic potential compared with nontumorigenic cancer cells and $50 \%$ of animals injected with as few as $100 \mathrm{CD} 44^{+} \mathrm{CD} 24^{+} \mathrm{ESA}^{+}$cells formed tumors that were histologically indistinguishable from the human tumors from which they originated. The enhanced ability of $\mathrm{CD} 44^{+} \mathrm{CD} 24^{+} \mathrm{ESA}^{+}$ pancreatic cancer cells to form tumors was confirmed by using an orthotopic pancreatic tail injection model. The $\mathrm{CD} 44^{+} \mathrm{CD} 24^{+} \mathrm{ESA}^{+}$pancreatic cancer cells showed the stem cell properties of self-renewal as well as the ability to produce differentiated progeny, with a further increase in the expression of the developmental signaling molecule sonic hedgehog.

GI malignancies are noted for their characteristically high incidence of disease relapse after surgery or chemotherapy as well as resistance to chemotherapy, all of which may be explained by the aggressive phenotype of their CSCs. To effectively combat GI cancers, specific markers for CSCs must be used to target these populations using novel therapeutic agents.

\section{Acknowledgements}

Grant Support: NIH RO1 CA106614A [18], NIH RO1 DK56111 [18], NIH RO1 CA4285718A [18], VA 
Merit Award [18], R. Robert and Sally D. Funderburg Research Scholar [18].

\section{References}

[1] C.M. Southam et al., Effect of leukocytes on transplantability of human cancer, Cancer 19(11) (1966), 1743-1753.

[2] D.E. Bergsagel and F.A. Valeriote, Growth characteristics of a mouse plasma cell tumor, Cancer Res 28(11) (1968), $2187-$ 2196.

[3] A.J. Salsbury, The significance of the circulating cancer cell, Cancer Treat Rev 2(1) (1975), 55-72.

[4] H.R. Schumacher et al., Acute leukemic cells. Qualitative and quantitative electron microscopy, Am J Pathol 73(1) (1973), 27-46.

[5] M. Shackleton et al., Generation of a functional mammary gland from a single stem cell, Nature 439(7072) (2006), 84-8.

[6] J. Stingl et al., Purification and unique properties of mammary epithelial stem cells, Nature 439(7079) (2006), 993-997.

[7] M. Brittan and N.A. Wright, Stem cell in gastrointestinal structure and neoplastic development, Gut 53(6) (2004), 899-910.

[8] S.P. Bach, A.G. Renehan and C.S. Potten, Stem cells: the intestinal stem cell as a paradigm, Carcinogenesis 21(3) (2000), 469-476.

[9] J.P. Seery, Stem cells of the oesophageal epithelium, J Cell Sci 115(Pt 9) (2002), 1783-1789.

[10] F.M. Watt, Stem cell fate and patterning in mammalian epidermis, Curr Opin Genet Dev 11(4) (2001), 410-417.

[11] E. Sancho, E. Batlle and H. Clevers, Live and let die in the intestinal epithelium, Curr Opin Cell Biol 15(6) (2003), 763770 .

[12] C.F. Kim et al., Identification of bronchioalveolar stem cells in normal lung and lung cancer, Cell 121(6) (2005), 823-835.

[13] C.E. Gargett, Stem cells in gynaecology, Aust N Z J Obstet Gynaecol 44(5) (2004), 380-386.

[14] C.S. Potten, Stem cells in gastrointestinal epithelium: numbers, characteristics and death, Philos Trans $R$ Soc Lond B Biol Sci 353(1370) (1998), 821-830.

[15] J.A. Schalken and G. van Leenders, Cellular and molecular biology of the prostate: stem cell biology, Urology 62(5 Suppl 1) (2003), 11-20.

[16] R. Galli et al., Isolation and characterization of tumorigenic, stem-like neural precursors from human glioblastoma, Cancer Res 64(19) (2004), 7011-7021.

[17] S.K. Singh et al., Identification of a cancer stem cell in human brain tumors, Cancer Res 63(18) (2003), 5821-5828.

[18] S.K. Singh et al., Identification of human brain tumour initiating cells, Nature 432(7015) (2004), 396-401.

[19] J. Miki et al., Identification of putative stem cell markers, CD133 and CXCR4, in hTERT-immortalized primary nonmalignant and malignant tumor-derived human prostate epithelial cell lines and in prostate cancer specimens, Cancer Res 67(7) (2007), 3153-3161.

[20] W.R. Otto, Lung epithelial stem cells, J Pathol 197(4) (2002), 527-535.

[21] A.L. Vescovi, R. Galli and B.A. Reynolds, Brain tumour stem cells, Nat Rev Cancer 6(6) (2006), 425-436.

[22] R.C. Haggitt and B.J. Reid, Hereditary gastrointestinal polyposis syndromes, Am J Surg Pathol 10(12) (1986), 871-887.

[23] M.T. Veeman, J.D. Axelrod and R.T. Moon, A second canon. Functions and mechanisms of beta-catenin-independent Wnt signaling, Dev Cell 5(3) (2003), 367-377.
[24] U. Gat et al., De Novo hair follicle morphogenesis and hair tumors in mice expressing a truncated beta-catenin in skin, Cell 95(5) (1998), 605-614.

[25] D. Pinto et al., Canonical Wnt signals are essential for homeostasis of the intestinal epithelium, Genes Dev 17(14) (2003), 1709-1713.

[26] R. Smits et al., Apc1638N: a mouse model for familial adenomatous polyposis-associated desmoid tumors and cutaneous cysts, Gastroenterology 114(2) (1998), 275-283.

[27] H. Fujie et al., Frequent beta-catenin aberration in human hepatocellular carcinoma, Hepatol Res 20(1) (2001), 39-51.

[28] S. Satoh et al., AXIN1 mutations in hepatocellular carcinomas, and growth suppression in cancer cells by virus-mediated transfer of AXIN1, Nat Genet 24(3) (2000), 245-250.

[29] T. Reya and H. Clevers, Wnt signalling in stem cells and cancer, Nature 434(7035) (2005), 843-850.

[30] X.H. Feng and R. Derynck, Specificity and versatility in tgfbeta signaling through Smads, Annu Rev Cell Dev Biol 21 (2005), 659-693.

[31] J. Massague, TGF-beta signal transduction, Annu Rev Biochem 67 (1998), 753-791.

[32] T. Tsukazaki et al., SARA, a FYVE domain protein that recruits Smad2 to the TGFbeta receptor, Cell 95(6) (1998), 779791.

[33] Y. Tang et al., Disruption of transforming growth factorbeta signaling in ELF beta-spectrin-deficient mice, Science 299(5606) (2003), 574-577.

[34] D. Bartsch et al., Higher frequency of DPC4/Smad4 alterations in pancreatic cancer cell lines than in primary pancreatic adenocarcinomas, Cancer Lett 139(1) (1999), 43-49.

[35] V. Katuri et al., Inactivation of ELF/TGF-beta signaling in human gastrointestinal cancer, Oncogene 24(54) (2005), 80128024.

[36] Y. Tang et al., Transforming growth factor-beta suppresses nonmetastatic colon cancer through Smad4 and adaptor protein ELF at an early stage of tumorigenesis, Cancer Res $\mathbf{6 5}(10)$ (2005), 4228-4237.

[37] V. Katuri et al., Critical interactions between TGF-beta signaling/ELF, and E-cadherin/beta-catenin mediated tumor suppression, Oncogene 25(13) (2006), 1871-1886.

[38] D. Liaw et al., Germline mutations of the PTEN gene in Cowden disease, an inherited breast and thyroid cancer syndrome, Nat Genet 16(1) (1997), 64-67.

[39] X.C. He et al., PTEN-deficient intestinal stem cells initiate intestinal polyposis, Nat Genet 39(2) (2007), 189-198.

[40] E. Labbe, A. Letamendia and L. Attisano, Association of Smads with lymphoid enhancer binding factor $1 /$ T cell-specific factor mediates cooperative signaling by the transforming growth factor-beta and wnt pathways, Proc Natl Acad Sci USA 97(15) (2000), 8358-8363.

41] M. Furuhashi et al., Axin facilitates Smad3 activation in the transforming growth factor beta signaling pathway, Mol Cell Biol 21(15) (2001), 5132-5141.

[42] D.J. Satterwhite and K.L. Neufeld, TGF-beta targets the Wnt pathway components, APC and beta-catenin, as Mv1Lu cells undergo cell cycle arrest, Cell Cycle 3(8) (2004), 1069-1073.

[43] S. Miraglia et al., A novel five-transmembrane hematopoietic stem cell antigen: isolation, characterization, and molecular cloning, Blood 90(12) (1997), 5013-5021.

[44] P.A. Horn et al., Expression of AC133, a novel hematopoietic precursor antigen, on acute myeloid leukemia cells, Blood 93(4) (1999), 1435-1437. 
[45] L. Ricci-Vitiani et al., Identification and expansion of human colon-cancer-initiating cells, Nature 445(7123) (2007), 111115 .

[46] C.A. O'Brien et al., A human colon cancer cell capable of initiating tumour growth in immunodeficient mice, Nature 445(7123) (2007), 106-110.

[47] C.E. Craig et al., The histopathology of regeneration in massive hepatic necrosis, Semin Liver Dis 24(1) (2004), 49-64.

[48] A. Suetsugu et al., Characterization of CD133+ hepatocellular carcinoma cells as cancer stem/progenitor cells, Biochem Biophys Res Commun 351(4) (2006), 820-824.
[49] N. Haraguchi et al., Characterization of a side population of cancer cells from human gastrointestinal system, Stem Cells 24(3) (2006), 506-513.

[50] A. Durnez et al., The clinicopathological and prognostic relevance of cytokeratin 7 and 19 expression in hepatocellular carcinoma. A possible progenitor cell origin, Histopathology 49(2) (2006), 138-151.

[51] C. Li et al., Identification of pancreatic cancer stem cells, Cancer Res 67(3) (2007), 1030-1037. 


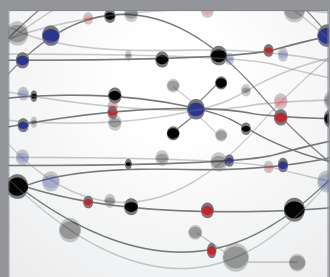

The Scientific World Journal
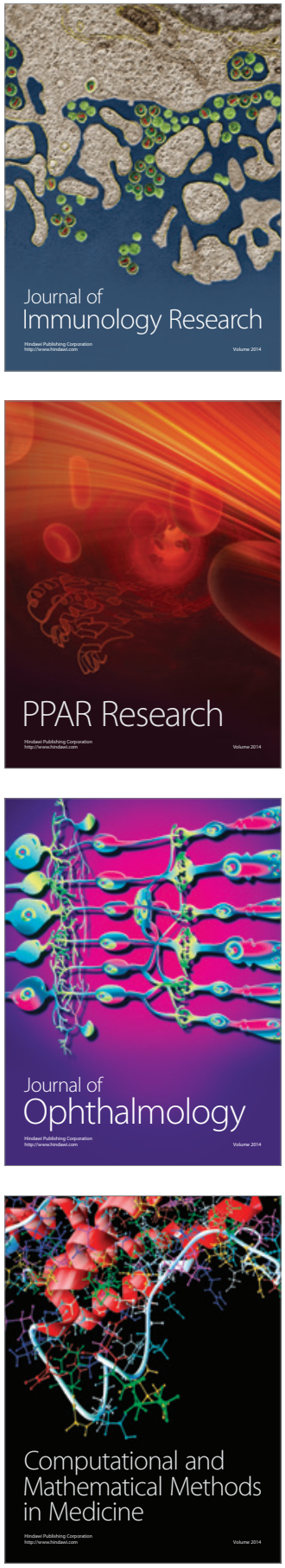

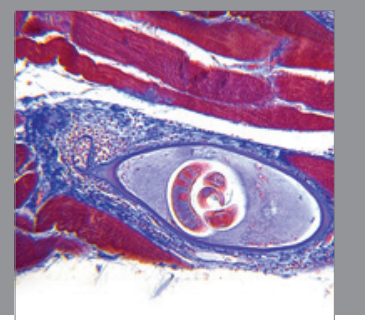

Gastroenterology

Research and Practice
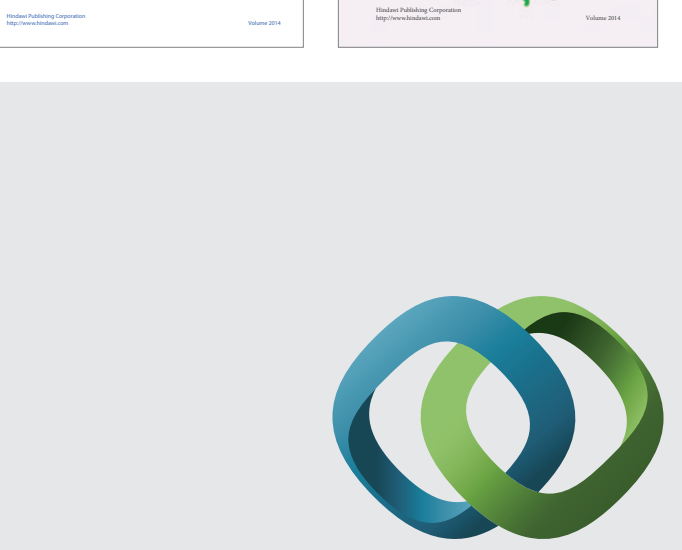

\section{Hindawi}

Submit your manuscripts at

http://www.hindawi.com
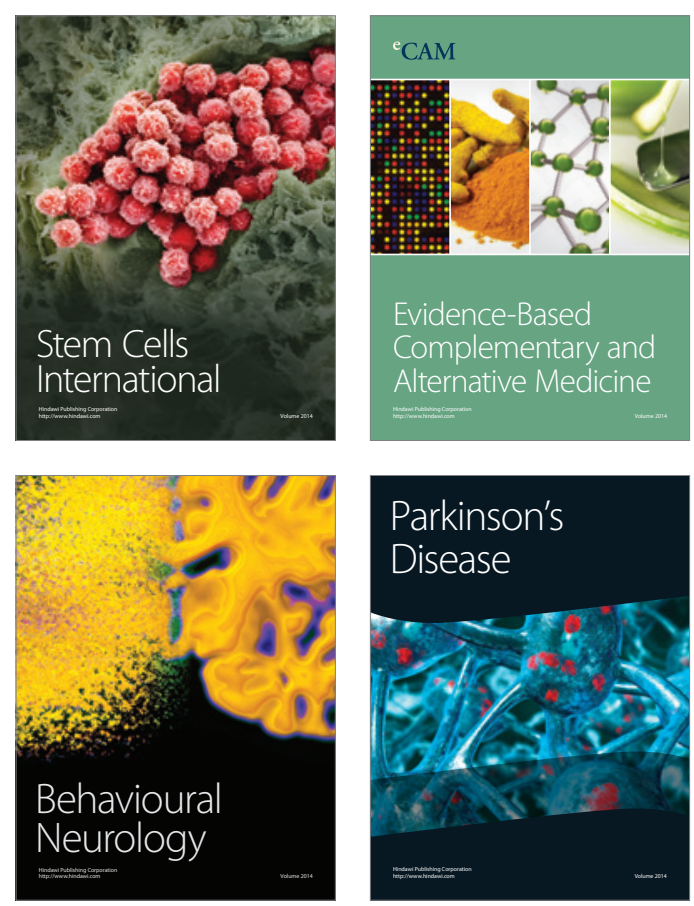

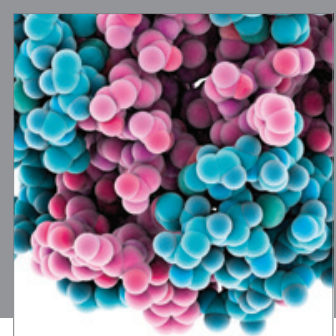

Journal of
Diabetes Research

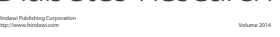

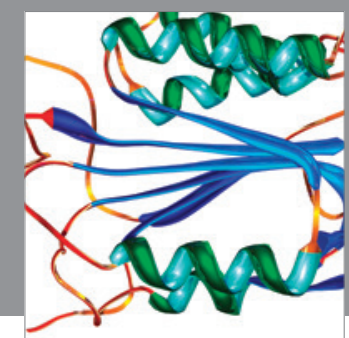

Disease Markers
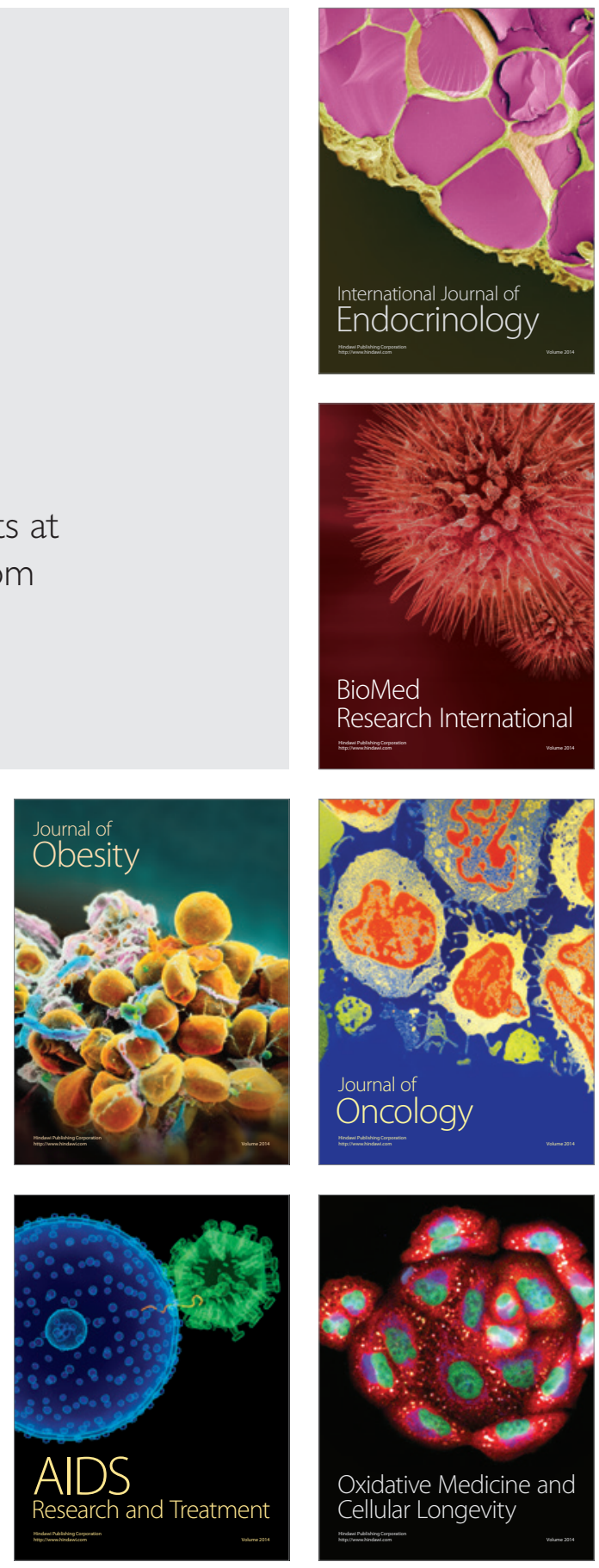